\title{
Variability in lutetium-177 SPECT quantification between different state-of- the-art SPECT/CT systems
}

Steffie M. B. Peters ${ }^{1 * \dagger} \mathbb{D}$, Sebastiaan L. Meyer Viol ${ }^{2 \dagger}$, Niels R. van der Werf ${ }^{3 \dagger}$, Nick de Jong ${ }^{4}$, Floris H. P. van Velden ${ }^{4}$, Antoi Meeuwis ${ }^{1}$, Mark W. Konijnenberg ${ }^{3}$, Martin Gotthardt', Hugo W. A. M. de Jong ${ }^{2}$ and Marcel Segbers ${ }^{3}$

* Correspondence: steffie.peters@
radboudumc.nl
†Steffie M. B. Peters, Sebastiaan L.
Meyer Viol and Niels R. van der
Werf contributed equally to this
work.
'Department of Radiology and
Nuclear Medicine, Department of
Radiology and Nuclear Medicine,
Radboud University Medical Center,
P.O. Box 9101,6500, HB, Nijmegen,
The Netherlands
Full list of author information is
available at the end of the article

* Correspondence: steffie.peters@ radboudumc.n Werf contributed equally to this work Nuclear Medicine, Department of Radiology and Nuclear Medicine, Radboud University Medical Center, The Netherland

available at the end of the article

\begin{abstract}
Background: Quantitative SPECT imaging in targeted radionuclide therapy with lutetium-177 holds great potential for individualized treatment based on dose assessment. The establishment of dose-effect relations requires a standardized method for SPECT quantification. The purpose of this multi-center study is to evaluate quantitative accuracy and inter-system variations of different SPECT/CT systems with corresponding commercially available quantitative reconstruction algorithms. This is an important step towards a vendor-independent standard for quantitative lutetium-177 SPECT.
\end{abstract}

Methods: Four state-of-the-art SPECT/CT systems were included: Discovery ${ }^{\mathrm{TM}} \mathrm{NM} /$ CT 670Pro (GE Healthcare), Symbia Intevo ${ }^{\mathrm{TM}}$, and two Symbia ${ }^{\mathrm{TM}}$ T16 (Siemens Healthineers). Quantitative accuracy and inter-system variations were evaluated by repeatedly scanning a cylindrical phantom with 6 spherical inserts $(0.5-113$ $\mathrm{ml}$ ). A sphere-to-background activity concentration ratio of 10:1 was used. Acquisition settings were standardized: medium energy collimator, body contour trajectory, photon energy window of $208 \mathrm{keV}( \pm 10 \%)$, adjacent $20 \%$ lower scatter window, $2 \times 64$ projections, $128 \times 128$ matrix size, and 40 s projection time. Reconstructions were performed using GE Evolution with Q.Metrix ${ }^{\mathrm{T}}$, Siemens xSPECT Quant ${ }^{T M}$, Siemens Broad Quantification ${ }^{\text {TM }}$ or Siemens Flash3D ${ }^{\text {TM }}$ algorithms using vendor recommended settings. In addition, projection data were reconstructed using Hermes SUV SPECT ${ }^{\mathrm{T}}$ with standardized reconstruction settings to obtain a vendor-neutral quantitative reconstruction for all systems. Volumes of interest (VOI) for the spheres were obtained by applying a 50\% threshold of the sphere maximum voxel value corrected for background activity. For each sphere, the mean and maximum recovery coefficient $\left(\mathrm{RC}_{\text {mean }}\right.$ and $\mathrm{RC} C_{\max }$ ) of three repeated measurements was calculated, defined as the imaged activity concentration divided by the actual activity concentration. Inter-system variations were defined as the range of RC over all systems,

Results: RC decreased with decreasing sphere volume. Inter-system variations with vendor-specific reconstructions were between 0.06 and 0.41 for $R C_{\text {mean }}$ depending on sphere size (maximum 118\% quantification difference), and improved to 0.02-0.19 with vendor-neutral reconstructions (maximum 38\% quantification difference).

(Continued on next page) 
(Continued from previous page)

Conclusion: This study shows that eliminating sources of possible variation drastically reduces inter-system variation in quantification. This means that absolute SPECT quantification for ${ }^{177} \mathrm{Lu}$ is feasible in a multi-center and multi-vendor setting; however, close agreement between vendors and sites is key for multi-center dosimetry and quantitative biomarker studies.

\section{Introduction}

Quantitative SPECT imaging in targeted radionuclide therapy with lutetium-177 $\left({ }^{177} \mathrm{Lu}\right)$ holds great potential for dosimetry-based individualized treatment and may improve prediction of therapy response, prevention of toxicity effects and treatment follow-up. With the advent of ${ }^{177}$ Lu-PSMA therapy [1-4], it is expected that dosimetry will play a pivotal role in the reliable determination of dose-response relationships in tumors. But also our understanding of biomarker studies and already well-established radionuclide therapies in neuroendocrine tumors [5-9] may profit from optimized quantitative SPECT imaging for sophisticated dosimetry.

SPECT quantification is considered less straightforward than PET quantification [10, 11]. This can be explained by several factors including lower sensitivity due to the necessary use of a collimator, the need for more complicated scatter and attenuation correction [11] and a lower resolution creating partial volume effects. Several studies investigated the quantitative performance of SPECT for a variety of radionuclides, including technetium$99 \mathrm{~m}\left({ }^{99 \mathrm{~m}} \mathrm{Tc}\right)[12,13]$, indium-111 $\left({ }^{111} \mathrm{In}\right)$ [14-16], iodine-131 $\left({ }^{131} \mathrm{I}\right)[17]$, yttrium-90 $\left({ }^{90} \mathrm{Y}\right)$, or a combination of these $[18,19]$ and concluded that quantification is possible, be it with certain limitations, for example, with regard to small structures as a result to the partial volume effect. Beauregard et al. looked into the quantitative accuracy of ${ }^{177} \mathrm{Lu}$ on one SPECT/CT system [20] and found that this could yield more accurate dosimetry estimates than planar imaging. Hippeläinen et al. compared the results of different ordered subset expectation maximization (OSEM) reconstruction algorithms [21] and concluded that alignment was best when the images were corrected for attenuation, scatter, and detector and collimator response. Various SPECT/CT vendors have responded to the increasing need for SPECT quantification and now commercially offer software packages for quantification of several radionuclides including ${ }^{177} \mathrm{Lu}[22-24]$.

However, standardization of protocols such that quantitative results can be reliably compared between systems requires more insight in their quantitative accuracy and performance. This is key for, e.g., multi-center research trials involving absolute SPECT quantification, especially those aimed towards dosimetry. Our previous study compared quantification for SPECT/CT systems from different vendors at different imaging centers for technetium-99 m and showed that standardizing reconstruction decreased inter-system variability [25]. The aim of this study is to extend these findings to ${ }^{177} \mathrm{Lu}$. The quantitative accuracy and inter-system variability of recovery coefficients (RC) were determined using phantom experiments and the effects of lesion volume and reconstruction algorithm on $\mathrm{RC}$ were investigated. The results of these comparisons can be used as input for a vendor-independent standard for absolute quantitative SPECT of ${ }^{177} \mathrm{Lu}$. 
Table 1 Main properties of the dual headed SPECT/CT systems used in this study

\begin{tabular}{|c|c|c|c|c|}
\hline System & $\begin{array}{l}\text { General Electric } \\
\text { Discovery NM/CT } \\
670 \text { Pro }\end{array}$ & $\begin{array}{l}\text { Siemens } \\
\text { Symbia Intevo Bold }\end{array}$ & $\begin{array}{l}\text { Siemens } \\
\text { Symbia T16 system } 1\end{array}$ & $\begin{array}{l}\text { Siemens } \\
\text { Symbia T16 system } 2\end{array}$ \\
\hline $\begin{array}{l}\text { Imaging } \\
\text { center }\end{array}$ & $\begin{array}{l}\text { Leiden University } \\
\text { Medical Center }\end{array}$ & $\begin{array}{l}\text { University Medical } \\
\text { Center Utrecht }\end{array}$ & $\begin{array}{l}\text { Radboud University } \\
\text { Medical Center }\end{array}$ & $\begin{array}{l}\text { Erasmus University } \\
\text { Medical Center }\end{array}$ \\
\hline $\begin{array}{l}\text { SPECT } \\
\text { detector }\end{array}$ & $\begin{array}{l}3 / 8 \text { " Nal crystal } \\
59 \mathrm{PMT}^{*} \\
40 \times 54 \mathrm{~cm} \mathrm{FOV}^{*}\end{array}$ & $\begin{array}{l}\text { 3/8" Nal crystal } \\
59 \text { PMT }^{*} \\
38.7 \times 53.3 \mathrm{~cm} \mathrm{FOV*}^{*}\end{array}$ & $\begin{array}{l}\text { 3/8" Nal crystal } \\
59 \text { PMT }^{*} \\
38.7 \times 53.3 \mathrm{~cm} \mathrm{FOV}^{*}\end{array}$ & $\begin{array}{l}\text { 3/8" Nal crystal } \\
59 \mathrm{PMT}^{*} \\
38.7 \times 53.3 \mathrm{~cm} \mathrm{FOV}^{*}\end{array}$ \\
\hline CT & 16-slice & 16-slice & 16-slice & 16-slice \\
\hline
\end{tabular}

*PMT photomultiplier tube, FOV field of view

\section{Methods}

\section{SPECT/CT systems}

Four SPECT/CT systems from two manufacturers were included in this study: a Discovery NM/CT 670 Pro (GE Healthcare, Milwaukee, USA), a Symbia Intevo Bold, and two Symbia T16's (Siemens Healthineers, Erlangen, Germany) (Table 1). Three out of four systems had commercial software packages for quantification installed, as listed in Table 2.

Table 2 Reconstruction/quantification parameters and processing software used in this study. Quantification packages Q. Metrix, xSPECT Quant, and Broad quantification enable quantitative reconstructions in the scanner software

\begin{tabular}{|c|c|c|c|c|c|}
\hline System & $\begin{array}{l}\text { Discovery NM/ } \\
\text { CT } 670 \text { Pro }\end{array}$ & $\begin{array}{l}\text { Symbia Intevo } \\
\text { Bold }\end{array}$ & $\begin{array}{l}\text { Symbia T16 } \\
\text { system } 1\end{array}$ & $\begin{array}{l}\text { Symbia T16 } \\
\text { system } 2\end{array}$ & $\begin{array}{l}\text { All } \\
\text { (standardized) }\end{array}$ \\
\hline Imaging center & $\begin{array}{l}\text { Leiden University } \\
\text { Medical Center }\end{array}$ & $\begin{array}{l}\text { University } \\
\text { Medical Center } \\
\text { Utrecht }\end{array}$ & $\begin{array}{l}\text { Radboud } \\
\text { University } \\
\text { Medical Center }\end{array}$ & $\begin{array}{l}\text { Erasmus } \\
\text { University } \\
\text { Medical Center }\end{array}$ & All \\
\hline Reconstruction & $\begin{array}{l}\text { OSEM }^{a}+ \\
\text { Evolution with } \\
\text { PSF }^{a} \text { correction }\end{array}$ & $\begin{array}{l}\text { WCG }^{a}+\times S P E C T \\
\text { with PSF } \\
\text { correction }\end{array}$ & $\begin{array}{l}\text { OSEM }^{a}+\text { Flash 3D } \\
\text { with PSF } \\
\text { correction }\end{array}$ & $\begin{array}{l}\text { OSEM }^{a}+\text { Hybrid } \\
\text { Recon V3.0.0 } \\
\text { with PSF } \\
\text { correction }\end{array}$ & $\begin{array}{l}\text { OSEM }^{\mathrm{a}}+\text { Hybrid } \\
\text { Recon V } 3.0 .0 \\
\text { with PSF } \\
\text { correction }\end{array}$ \\
\hline Quantification & Q.Metrix & $\begin{array}{l}\text { xSPECT Quant/ } \\
\text { Broad } \\
\text { Quantificationb }^{\text {bu }}\end{array}$ & Manual analysis & $\begin{array}{l}\text { Hermes SUV } \\
\text { SPECT }\end{array}$ & $\begin{array}{l}\text { Hermes SUV } \\
\text { SPECT }\end{array}$ \\
\hline Iterations & $9^{d}$ & 6 & 4 & 5 & 5 \\
\hline Subsets & 10 & 8 & 8 & 16 & 16 \\
\hline $\begin{array}{l}\text { Post- } \\
\text { reconstruction } \\
\text { filter }\end{array}$ & None & 5 mm (Gaussian) & 4 mm (Gaussian) & 5 mm (Gaussian) & 5 mm (Gaussian) \\
\hline $\begin{array}{l}\text { Attenuation } \\
\text { correction }\end{array}$ & CT based $^{c}$ & CT based $^{c}$ & CT based $^{c}$ & CT based $^{c}$ & $C T$ based $^{c}$ \\
\hline $\begin{array}{l}\text { Scatter } \\
\text { Correction }\end{array}$ & $\mathrm{DEW}^{\mathrm{a}}(170 \pm 10 \%)$ & $\mathrm{DEW}^{\mathrm{a}}(170 \pm 10 \%)$ & $\mathrm{DEW}^{\mathrm{a}}(170 \pm 10 \%)$ & $\begin{array}{l}\text { Monte } \\
\text { Carlo-based }\end{array}$ & $\begin{array}{l}\text { Monte } \\
\text { Carlo-based }\end{array}$ \\
\hline $\begin{array}{l}\text { Reconstruction } \\
\text { voxel size }\end{array}$ & $\begin{array}{l}2.0 \times 2.0 \times 2.0 \\
\mathrm{~mm}^{3} \mathrm{e}\end{array}$ & $\begin{array}{l}4.9 \times 4.9 \times 4.9 \\
\mathrm{~mm}^{3}\end{array}$ & $\begin{array}{l}4.8 \times 4.8 \times 4.8 \\
\mathrm{~mm}^{3}\end{array}$ & $\begin{array}{l}4.8 \times 4.8 \times 4.8 \\
\mathrm{~mm}^{3}\end{array}$ & $\begin{array}{l}4.8 \times 4.8 \times 4.8 \\
\mathrm{~mm}^{3}\end{array}$ \\
\hline
\end{tabular}

${ }^{a}$ OSEM ordered subset expectation maximization; WCG weighted conjugate gradient, PSF point spread function, DEW double energy window

${ }^{\mathrm{b}}$ For XSPECT Quant and Broad quantification separate measurements were performed

c Bilinear conversion of $\mathrm{HU}$ into attenuation coefficients at $208 \mathrm{keV}$

d Settings were not according to vendor recommendation but to the literature [13]

e Interpolation to $2.0 \times 2.0 \times 2.0 \mathrm{~mm}^{3}$ voxels by $\mathrm{Q}$. Metrix for quantification, as recommended by the vendor 


\section{Phantom}

A modified cylindrical Jaszczak phantom (Fig. 1) was used, with a background compartment volume of approximately $6.7 \mathrm{l}$ and regular inserts replaced by 6 spherical inserts representing various lesion sizes with inner diameters (and brief volumes) of $9.9 \mathrm{~mm}(0.5 \mathrm{ml}), 15.4 \mathrm{~mm}(2.0 \mathrm{ml})$, $19.8 \mathrm{~mm}$ (4.0 ml), $24.8 \mathrm{~mm}(8.0 \mathrm{ml}), 31.3 \mathrm{~mm}(16.0 \mathrm{ml})$, and $60.0 \mathrm{~mm}(113 \mathrm{ml})$.

The spheres and background compartment were filled with a homogeneous solution of ${ }^{177} \mathrm{Lu}$ in water with an activity concentration of approximately $750 \mathrm{kBq} / \mathrm{ml}$ for the spheres and $75 \mathrm{kBq} / \mathrm{ml}$ for the background compartment, resulting in a sphere-to-background ratio of 10:1. The concentrations were based upon the expected lesion uptake in lutetium therapy [2, 26]. The solution was saturated with a $50 \mathrm{mM}$ ethylenediaminetetraacetic acid (EDTA) solution to prevent precipitation of lutetium. Enabled by the long half-life time of ${ }^{177} \mathrm{Lu}\left(T_{1 / 2}=\right.$ 665 days), user preparation differences were excluded by the study set-up, as the phantom was filled once before being sent around to the participating centers. The time between first and last measurement was $74.7 \mathrm{~h}$, and during acquisition, the measurement time per angle was adjusted for activity decay to obtain similar count statistics for each measurement.

To check for possible quantification differences caused by the use of different dose calibrators, a syringe filled with the same solution with an activity of $29.0 \mathrm{MBq}{ }^{177} \mathrm{Lu}$ was measured in each center and compared to the activity measured in the reference center.

\section{Acquisition}

Images were acquired with a Medium Energy General Purpose (MEGP) or Medium Energy Low Penetration (MELP) collimator (Additional file 1: Table S1). Acquisition settings were harmonized across all systems according to MIRD Pamphlet No. 26 [27]: body contour trajectory, a photon energy window of $208 \mathrm{keV}( \pm 10 \%)$, adjacent $20 \%$ lower scatter window, $2 \times 64$ projections, a $128 \times 128$ matrix size, and a reference projection time of $40 \mathrm{~s}$. On each system, the measurement was repeated three times to assess repeatability.

\section{Reconstruction}

Reconstructions were performed with vendor/center specific 3D iterative reconstruction algorithms and quantification packages (Table 2). The reconstructions were performed with scatter correction, CT-based attenuation correction (Additional file 1: Table S2) and resolution recovery, using vendor recommended settings (Table 2). In addition to vendor/center specific reconstructions, all raw data were reconstructed with a vendor neutral-reconstruction
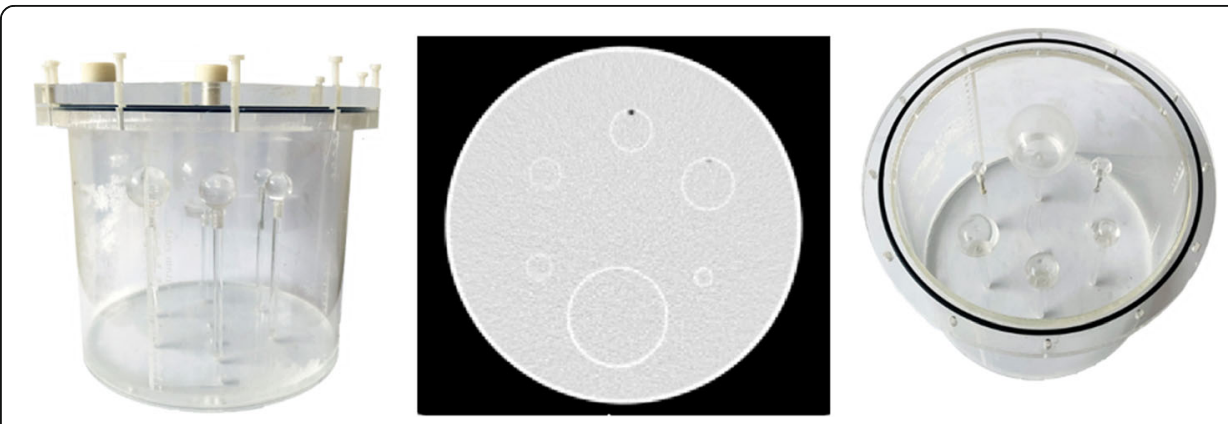

Fig. 1 The phantom used to determine the recovery coefficients. The second image is a CT slice of the phantom on which the order of the spheres can be seen 
algorithm (Hybrid Recon v3.0.0, Hermes SUV SPECT ${ }^{\mathrm{tm}}$, Stockholm, Sweden), with standardized reconstruction settings (Table 2) to obtain vendor-neutral quantitative reconstructions for all systems.

\section{Calibration factor}

All SPECT/CT systems were cross-calibrated for ${ }^{177} \mathrm{Lu}$ with the in-house dose calibrator according to the manufacturer's guidelines or to the center's standard practice (Additional file 1: Table S3). An exception was the Symbia Intevo Bold (xSPECT) quantification calibration, which is different from the other calibration methods, as it makes use of a (by manufacturer) included ${ }^{75} \mathrm{Se}$ calibrated sensitivity precision source instead of a ${ }^{177} \mathrm{Lu}$ source. All dose calibrators used in this study to cross-calibrate the SPECT systems undergo regular quality control according to national guidelines [28].

To determine the calibration factor for the vendor-neutral quantification method each site performed a calibration according to the guidelines of this particular software vendor. Each site scanned a homogeneous cylindrical phantom with a 6 to 71 volume and approximately $500 \mathrm{MBq}{ }^{177} \mathrm{Lu}$ with the same acquisition protocol as used in the experiments. Volumes of interest (VOIs) were drawn to obtain a calibration factor (CF):

$$
\mathrm{CF}\left[\frac{\frac{\mathrm{cps}}{\mathrm{ml}}}{\frac{\mathrm{kBq}}{\mathrm{ml}}}\right]=\frac{\left(\frac{\mu}{t \cdot n \cdot v}\right)}{C},
$$

where $\mu$ is the mean voxel value in counts in the reconstructed image, $t$ is the time per projection, $n$ is the number of projections, $v$ is the voxel size and $C$ is the actual activity concentration in the phantom.

\section{Image analysis}

Image analyses were performed using in-house developed software in Python. This script uses the SimpleITK toolkit region growing algorithm to determine the sphere VOI $[29,30]$. The VOIs were obtained by applying a $50 \%$ threshold of the sphere maximum voxel value with a correction for the background activity [31]:

$$
\mathrm{VOI}_{\text {thresh }, j}=0.5 \cdot\left(\mathrm{VOI}_{\text {max }, j}+\mathrm{VOI}_{\text {mean }, b g}\right)
$$

where $\mathrm{VOI}_{\text {thresh }, j}$ is the $\mathrm{VOI}$ threshold voxel value of sphere $j, \mathrm{VOI}_{\text {max, }}$ is the maximum voxel value in the sphere VOI and $\mathrm{VOI}_{\text {mean,bg }}$ is the mean voxel value in the background VOI. The background was determined by placing a single cylindrical VOI (diameter $9 \mathrm{~cm}, 5 \mathrm{~cm}$ height) in a uniform region within the phantom. For each sphere VOI, the mean and maximum recovery coefficient (respectively $\mathrm{RC}_{\text {mean }}$ and $\mathrm{RC}_{\max }$ ) were calculated, defined as the mean/maximum imaged activity concentration $\left(A_{i}\right)$ over the three consecutive measurements, divided by the actual activity concentration $\left(A_{a}\right)$ :

$$
\begin{aligned}
\mathrm{RC}_{\text {mean }, j} & =\frac{A_{i, j}}{A_{a, j}} \\
\mathrm{RC}_{\text {max }, j} & =\frac{A_{i, \text { max }, j}}{A_{a, j}}
\end{aligned}
$$

The inter-system variability was assessed for each sphere diameter by the range of the RC over all systems according to: 


$$
\text { Range }_{j}=\mathrm{RC}_{j, \max }-\mathrm{RC}_{j, \min }
$$

where $j$ is the sphere diameter. This range was calculated for both the $\mathrm{RC}_{\text {mean }}$ and $\mathrm{RC}_{\max }$.

This study included three systems of one vendor (Siemens), all consisting of (almost) equal hardware. Therefore, it was possible to compare quantification differences within one vendor as well, thereby focusing on differences between systems without the influence of their hardware.

\section{Error analysis}

Uncertainties were determined for both the CF and de RC according to the EANM guidelines by Gear et al. [32]. The recovery coefficient curve as a function of sphere volume was fitted with a 3-parameter logistic function.

\section{Results}

\section{Calibration}

Differences in activity in the syringe as determined by the dose calibrator in each center were $1-4 \%$ (Table 3 ).

Table 4 shows the calibration factors of each system. The error in CF is assumed to be within $5 \%$ since it is dominated by the uncertainty in the activity used in the cylindrical phantom. The activity was measured in a dose calibrator with an uncertainty smaller than 5\% (Table 3). In addition, the standard deviation in repeated measurements of a 389 voxel-sized VOI used for the average voxel counts $\mu$ (Eq. 1) was $0.7 \%$.

\section{Recovery coefficient}

The actual sphere-to-background activity concentration ratio based on dose calibrator measurements was 9.4:1. The median recovery coefficient of the background compartment for the five different vendor-specific reconstructions was 0.97 (range 0.92-1.06).

The center-specific SPECT reconstructions are shown in Fig. 2. Due to the low contrast compared to the background, the smallest sphere $(9.9 \mathrm{~mm}$ diameter $)$ is not or barely visible. The recovery coefficients of the spheres (Fig. 3a-e) decreased with decreasing sphere diameter on all systems. The variability between systems for $\mathrm{RC}_{\text {mean }}$ is visualized in Fig. $3 \mathrm{f}$ by plotting median and range for all systems. A large variability was found for spheres with a diameter $\leq 24.8 \mathrm{~mm}$ with a total $\mathrm{RC}$ range of up to 0.41 for (resulting in $118 \%$ quantification differences between systems) and 0.62 for $\mathrm{RC}_{\max }$ (139\% quantification differences), especially when compared to the largest sphere diameter $(60 \mathrm{~mm})$ that showed variability of $0.10(11 \%)$ and $0.19(15 \%)$ for $\mathrm{RC}_{\text {mean }}$ and $\mathrm{RC}_{\max }$ respectively.

Table 3 Measured differences in activity of a syringe filled with $29.0 \mathrm{MBq}{ }^{177} \mathrm{Lu}$ resulting from the use of different dose calibrators as used for the given systems

\begin{tabular}{ll}
\hline System & Deviation (compared to reference) \\
\hline Symbia T16 system 1 (reference) & 1.00 \\
Discovery NM/CT 670 Pro & 0.96 \\
Symbia Intevo Bold, xSPECT Quant & 1.01 \\
Symbia T16 system 2 & 0.99 \\
\hline
\end{tabular}


Table 4 Calibration factor (CF) for each system for vendor-neutral quantification

\begin{tabular}{ll}
\hline System & CF for Hermes SUV SPECT [cps/MBq] \\
\hline Discovery NM/CT 670 Pro & 6.2 \\
Symbia Intevo Bold, xSPECT Quant & 10.2 \\
Symbia Intevo Bold, Broad Quantification & 10.2 \\
Symbia T16 system 1 & 10.3 \\
Symbia T16 system 2 & 10.1 \\
\hline
\end{tabular}

The RC values were fitted with a 3-parameter logistic function as suggested in the EANM guidelines [32]. The additional parameter was introduced to allow the logistic function to reach asymptotic values different from unity. All curves showed a good correlation with the data (Pearson $R^{2}>0.96$ ).

\section{Effect of reconstruction algorithm on recovery coefficients}

The vendor-neutral SPECT reconstructions are shown in Fig. 4. Using this reconstruction algorithm on all data leads to visually more similar reconstructions. The median recovery coefficient of the background compartment for the four different vendorspecific reconstructions was 1.03 (range 0.91-1.07).

Figure 5 shows the recovery coefficient per system for the vendor-neutral reconstructions. By comparing Fig. 3 (vendor-specific) to Fig. 5 (vendor-neutral), a decrease in intersystem variability can be seen. This was confirmed by a large decrease in range for all sphere diameters $\leq 24.8 \mathrm{~mm}$ for both $\mathrm{RC}_{\text {mean }}$ ( 0.9 to 0.11 , resulting in quantification differences between systems of up to $38 \%$ ) and $\mathrm{RC}_{\max }(0.12$ to 0.17 , resulting in quantification differences of up to 46\%) (Fig. 5). Figure 6 shows the inter-system variability (RC range)

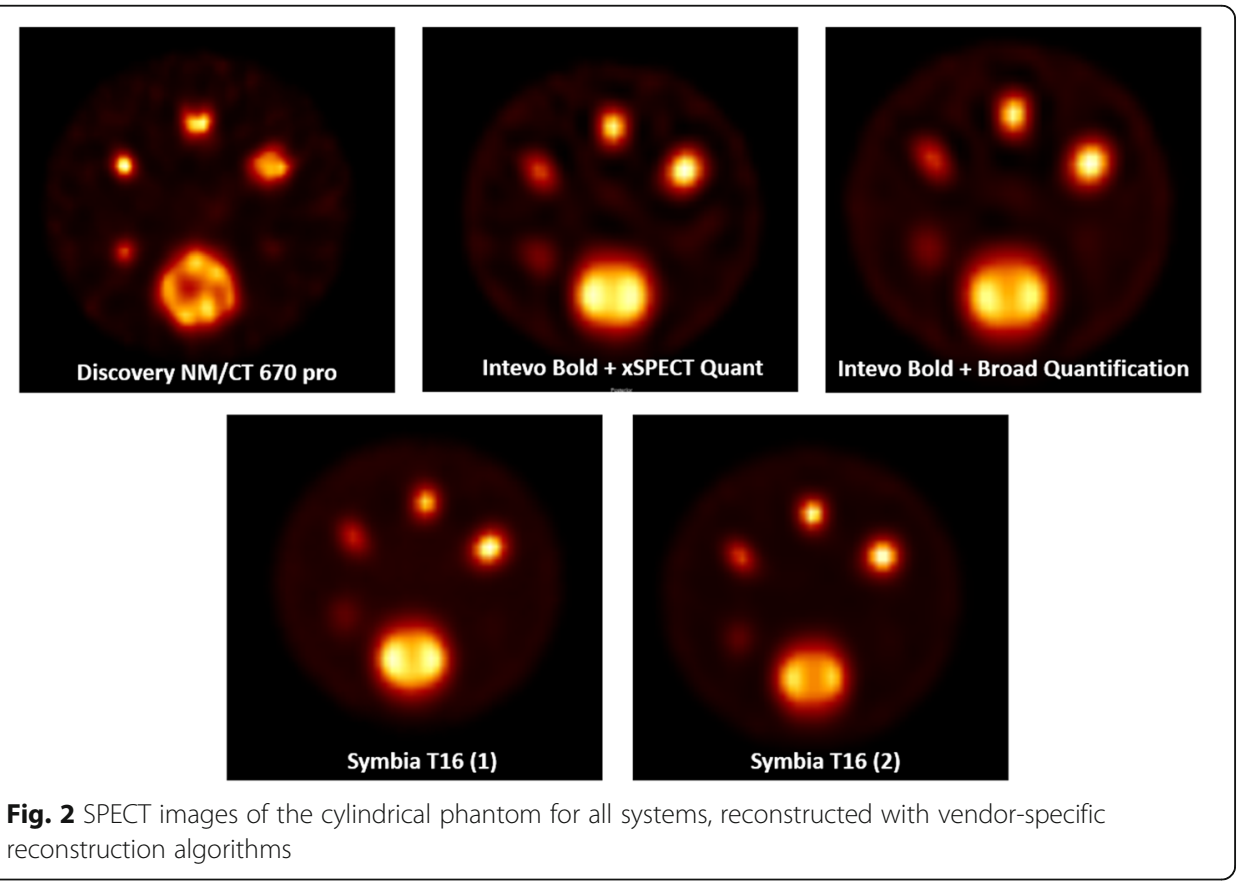



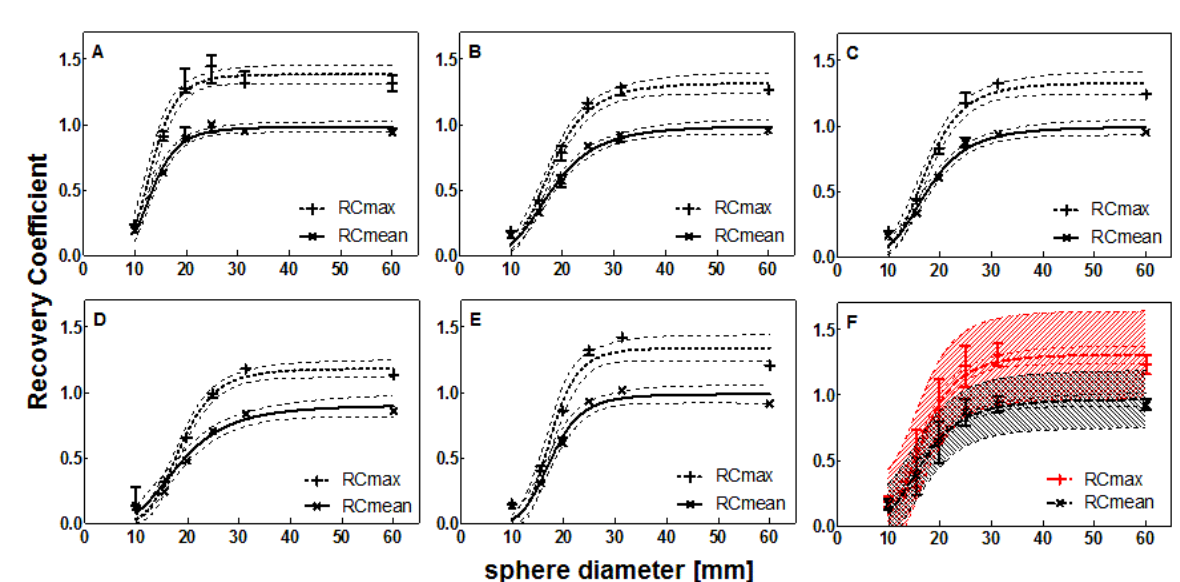

Fig. 3 Recovery coefficient as a function of sphere diameter for all systems separately (a-e) and for all systems combined (f), for data reconstructed with a vendor-specific algorithm. Median and range of three repetitive measurements per system. a Discovery NM/CT 670 Pro. b Symbia Intevo Bold with xSPECT Quant. c Symbia Intevo Bold with Broad Quantification. d Symbia T16 system 1. e Symbia T16 system 2. f Mean and standard deviation. All data were fitted with a 3-parameter logistic function (dashed line: 95\% Cl), for the combined data (f) also the $95 \%$ prediction interval is indicated (dashed area)

for vendor-neutral and vendor-specific reconstructions. For the two largest spheres, the inter-system variability slightly increased compared to the vendor-specific reconstruction, resulting in quantification differences of up to $21 \%$. When comparing systems within the same vendor, the inter-system variations result in quantification differences for all sphere sizes of up to $11 \%$ for $\mathrm{RC}_{\text {mean, }}$ and $12 \%$ for $\mathrm{RC}_{\max }$. This illustrates the large effect of the system hardware on quantification differences.

\section{Discussion}

This study shows that standardizing reconstruction settings decreases inter-system variability for quantification of ${ }^{177} \mathrm{Lu}$. This has important implications for multi-center studies where quantification plays an important role in dosimetry studies.

In order to eliminate preparation differences, the phantom was prepared only once and sent around to all participating centers. Furthermore, differences in activity measurements were monitored by measuring a syringe filled with a known amount of ${ }^{177} \mathrm{Lu}$ at each center and using the first center as a reference. Since one of the purposes of our study was to illustrate the differences in quantification between centers, the measured activity differences were not corrected to determine the RC. However, it was found that for one center, the deviation in activity was $4 \%$, which of course could also
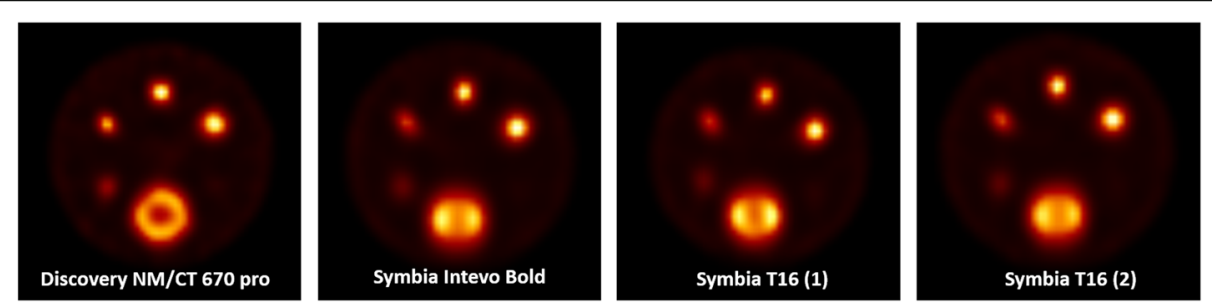

Fig. 4 SPECT images of the cylindrical phantom for all systems, reconstructed with a vendor-neutral algorithm 


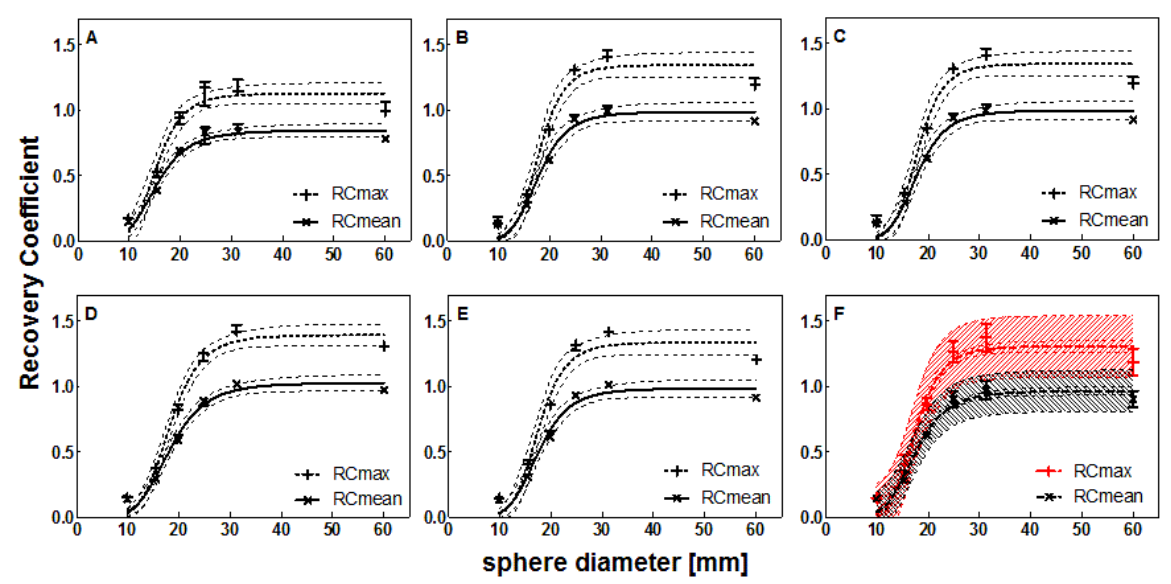

Fig. 5 Recovery coefficient as a function of sphere diameter for all systems separately (a-e) and for all systems combined (f), for data reconstructed with a vendor-neutral algorithm. Median and range of three repetitive measurements per system. a Discovery NM/CT 670 Pro. b Symbia Intevo Bold with XSPECT Quant. c Symbia Intevo Bold with Broad Quantification. d Symbia T16 system 1. e Symbia T16 system 2. f Mean and standard deviation for all systems combined. All data were fitted with a 3-parameter logistic function (dashed line $95 \% \mathrm{Cl}$ ), for the combined data (f) also the 95\% prediction interval is indicated (dashed area)

affect the final quantification on the SPECT/CT system since the cross-calibration for ${ }^{177} \mathrm{Lu}$ used for quantification was based on measurements on the center's own dose calibrator.

An adaptation of the Jaszczak phantom was used to determine the RC for different sphere diameters. The varying sphere diameters represent different lesion sizes and can give an estimate of the expected RC values for these lesions. For quantification as input for dosimetry, one could consider using a correction factor for smaller lesions, which show $R C$ values well below a value of 1.0 [32].

In this study, reconstruction settings were used that are applied in clinical practice for each center. These reconstruction settings were based mainly on the recommendations of the vendor, with possible adjustments by the center itself. These settings might

RCmean

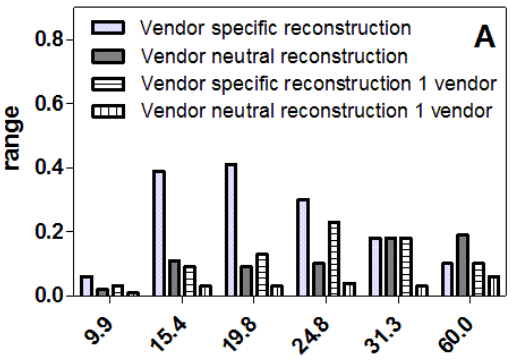

RCmax

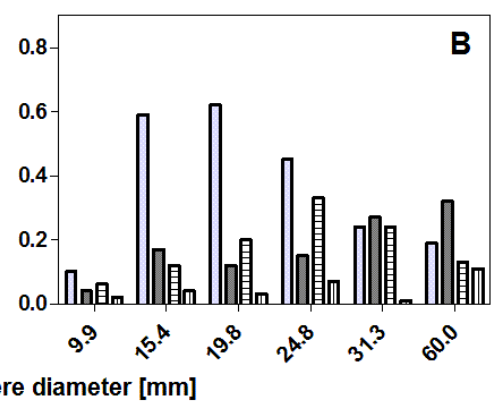

Fig. 6 Comparison in range over all systems in $\mathrm{RC}_{\text {mean }}(\mathbf{a})$ and $\mathrm{RC}$ max $(\mathbf{b})$ per sphere diameter for data reconstructed with a vendor-specific algorithm versus a vendor-neutral algorithm. Third and fourth columns give the same information but for systems of only one vendor, thus consisting of equal system hardware 
not be ideal for ${ }^{177} \mathrm{Lu}$ quantification, and the number of iterations used in reconstruction, as well as the possible additional use of a post-reconstruction filter, might influence the quantification [33, 34]. Furthermore, Dewaraja and colleagues [35] pointed out that post-reconstruction filtering is not desirable for quantifying total target activity, but acceptable when calculating $3 \mathrm{D}$ doses such as dose-volume histograms. This indicates that the reconstruction and post-reconstruction settings used in this study could be optimized further for quantification, possibly leading to better alignment in quantification between different centers and systems.

Due to the low contrast compared to the background, the smallest sphere $(9.9 \mathrm{~mm}$ diameter) was barely visible. This limitation in system spatial resolution is in line with the difficulty in quantification found by other studies $[18,19]$. Therefore, the recovery coefficients for this sphere volume should be interpreted with caution. Although it was shown that the range in $\mathrm{RC}$ between systems decreased from the second-smallest to the smallest sphere, it is expected that this is mainly due to the fact that the used segmentation method, a threshold based on $50 \%$ of the maximum voxel value, was not able to delineate a reliable VOI for the smallest sphere.

Image analysis was performed using an automated Python script that uses a background-corrected 50\% isocontour method based on the study of Frings and colleagues. Although a $42 \%$ isocontour method shows good recovery for both PET [36] and SPECT [13], the background-corrected 50\% isocontour method was chosen because of its high repeatability for PET in a multicenter settings [37] and to align our results as much as possible with the already existing standards for PET quantification [38]. However, a recently published study by Ryu et al. [39] showed that the line profiles over active spheres of reconstructed SPECT images (using ${ }^{99 \mathrm{~m}} \mathrm{Tc}$ and ${ }^{177} \mathrm{Lu}$ ) showed a very different profile than the same spheres measured on PET (using ${ }^{18} \mathrm{~F}$ and ${ }^{68} \mathrm{Ga}$ ). This indicates that a $50 \%$ isocontour method might not be the most ideal solution threshold for contouring in SPECT and that a lower threshold might be more appropriate as demonstrated by Collarino et al. [13]. However, the goal of this study was to assess the effects of different quantitative SPECT imaging systems, independent of the applied delineation method.

On the largest sphere, a low recovery coefficient was found, especially for reconstruction with the vendor-neutral algorithm (Hermes SUV SPECT). This can most probably be explained by the use of a $50 \%$ threshold for the VOI delineation, and a contribution of the Gibbs artifact, which is clearly visible in Figs. 2 and 4. A typical strategy in handling Gibbs ringing artifacts is to reduce them with compromised resolution [40, 41]. This reduction can be achieved by blurring the input image so that the data do not contain high-frequency components, by reconstruction other than PSF or by using post-reconstruction Gaussian filtering. Although not the goal of this study, reducing the Gibbs artifact might contribute to better alignment in quantification between centers. Additionally, for the Discovery NM/ CT 670 Pro and the Symbia T16 system 1, the low RC for the largest sphere might be explained by the number of iterations and subsets in the reconstruction settings. This was according to the center's standard settings which are based on vendor recommendations and other literature on reconstruction settings [13]. However, increasing the number of iterations might increase recovery and thereby improve quantification accuracy. 
The values for $\mathrm{RC}_{\max }$ are systematically over 1 for spheres with a diameter $>24.8 \mathrm{~mm}$. Although an overshoot for $\mathrm{RC}_{\max }$ was also found in other studies [13, 42], it was even higher in this study (1.3 \pm 0.2$)$. This overshoot is not a statistical error but is most probably the result of the resolution recovery algorithm that was used during reconstruction. This algorithm was used in the standard reconstruction as recommended by the vendor.

For spheres with a diameter $<25 \mathrm{~mm}$, RC quickly decreases as expected, mainly as a result of partial volume effects. For GE Evolution reconstruction, no postreconstruction Gaussian smoothing filter was applied, which might explain a higher RC for small sphere diameters. This high RC is also reflected in the large inter-system variations for small sphere diameters.

In this study, both $\mathrm{RC}_{\text {mean }}$ and $\mathrm{RC}_{\max }$ were determined and compared between systems. The results could be used to work towards a normalization between centers and systems. Depending on the application, the choice for either $\mathrm{RC}_{\text {mean }}$ or $\mathrm{RC}_{\max }$ as a tool for standardization could be more applicable. For example in ${ }^{18}$ F-FDG PET quantification, evaluation of treatment response is of main interest. In this case, this could be evaluated by using the $\mathrm{SUV}_{\max }$ and therefore standardization based on $\mathrm{RC}_{\max }$ would be a logical choice. For quantification of ${ }^{177} \mathrm{Lu}$, however, the most obvious application would be for the use in dosimetry for radionuclide therapies with ligands such as ${ }^{177} \mathrm{Lu}$-PSMA for prostate cancer or ${ }^{177} \mathrm{Lu}$-DOTATATE/DOTATOC for neuroendocrine tumors. This means a VOI is needed to determine the accumulated activity, in which the mean voxel value is the most relevant parameter. Therefore, we suggest that for normalization of ${ }^{177} \mathrm{Lu}$ quantification, the $\mathrm{RC}_{\text {mean }}$ could be used as a tool for standardization between centers.

Although this study provides valuable insight in quantification differences between systems, it only compared four SPECT/CT systems (five quantification methods). For one vendor, three systems and four quantification methods were included in this study. We showed that by eliminating the effect of system hardware, the inter-system variability was greatly reduced. Standardizing the reconstruction algorithm led to a further decrease in intersystem variability. It is therefore paramount to harmonize SPECT/CT image reconstructions in a multi-center/multi-vendor setting. These data can be used as input to work towards a standard for quantification of ${ }^{177} \mathrm{Lu}$, but it needs to be expanded to more centers and/or systems, preferably also across borders. We suggest that further standardization could improve the alignment of quantification between different SPECT/CT systems, comparable to the EARL accreditation program for ${ }^{18} \mathrm{~F}-\mathrm{FDG}$ PET/ CT [42]. Still, it is important to realize that variability in quantification between SPECT/CT systems will probably be larger than those found in this study, due to, for example, patient positioning and patient size.

\section{Conclusion}

This study shows that absolute SPECT quantification for ${ }^{177} \mathrm{Lu}$ is feasible in a multicenter and multi-vendor setting. With standardized acquisition protocols but centerspecific data reconstruction algorithms, the inter-system variability (range in RC between systems) was as large as 0.41 and 0.62 for $\mathrm{RC}_{\text {mean }}$ and $\mathrm{RC}_{\max }$, respectively. Standardizing reconstruction decreased this range to 0.19 and 0.32 , respectively. Close agreement between vendors and sites is key for multi-center dosimetry and quantitative biomarker studies. This study serves as an important step towards a vendorindependent standard for absolute quantification in SPECT/CT of ${ }^{177} \mathrm{Lu}$. 


\section{Supplementary information}

Supplementary information accompanies this paper at https://doi.org/10.1186/s40658-020-0278-3.

Additional file 1: Table S1. Characteristics of ME collimators for all used SPECT/CT systems. Table S2. Acquisition settings of low dose CT protocols used for attenuation correction. Table S3. Cross-calibration protocols for dose calibrators to SPECT/CT system.

\section{Acknowledgements}

The authors would like to thank Roel Wierts and Casper Beijst for their contribution to the discussion on the design of this study.

\section{Authors' contributions}

All the authors were involved in the experimental design and analysis and interpretation of the data. SMBP and SLMV took the lead in writing this manuscript. SMBP and AM performed measurements for Radboud University Medical Center. SLMV performed measurements for University Medical Center Utrecht. NRW, MS, and MK performed measurements for Erasmus Medical Center. Additionally, MS was responsible for writing the Python code. FHPV and NJ performed measurements for Leiden University Medical Center. All authors were involved in writing and reviewing the manuscript, and they all read and approved the final manuscript.

\section{Funding}

The research leading to these results has not received any external funding.

\section{Availability of data and materials}

The datasets used and/or analyzed during the current study are available from the corresponding author on reasonable request.

\section{Ethics approval and consent to participate}

Not applicable

\section{Consent for publication}

Not applicable

\section{Competing interests}

The authors declare that they have no competing interests.

\section{Author details}

${ }^{1}$ Department of Radiology and Nuclear Medicine, Department of Radiology and Nuclear Medicine, Radboud University Medical Center, P.O. Box 9101, 6500, HB, Nijmegen, The Netherlands. ²Department of Radiology and Nuclear Medicine, University Medical Center Utrecht, Utrecht, The Netherlands. ${ }^{3}$ Department of Radiology and Nuclear Medicine, Erasmus MC, Rotterdam, The Netherlands. ${ }^{4}$ Department of Radiology, Section of Medical Technology, Leiden University Medical Center, Leiden, The Netherlands.

Received: 7 October 2019 Accepted: 27 January 2020

Published online: 11 February 2020

\section{References}

1. Ahmadzadehfar H, Rahbar K, Kürpig S, Bögemann M, Claesener M, Eppard E, et al. Early side effects and first results of radioligand therapy with 177 Lu-DKFZ-617 PSMA of castrate-resistant metastatic prostate cancer: a two-Centre study. EJNMMI Res. 2015;5(1):36.

2. Delker A, Fendler WP, Kratochwil C, Brunegraf A, Gosewisch A, Gildehaus FJ, et al. Dosimetry for 177 Lu-DKFZPSMA-617: a new radiopharmaceutical for the treatment of metastatic prostate cancer. Eur J Nucl Med Mol Imaging. 2016:43(1):42-51.

3. Kabasakal L, AbuQbeitah M, Aygün A, Yeyin N, Ocak M, Demirci E, et al. Pre-therapeutic dosimetry of normal organs and tissues of 177 Lu-PSMA-617 prostate-specific membrane antigen (PSMA) inhibitor in patients with castration-resistant prostate cancer. Eur J Nucl Med Mol Imaging. 2015;42(13):1976-83.

4. Kratochwil C, Giesel FL, Stefanova M, Benesova M, Bronzel M, Afshar-Oromieh A, et al. PSMA-targeted radionuclide therapy of metastatic castration-resistant prostate cancer with Lu-177 labeled PSMA-617. J Nucl Med. 2016;57(8):1170-6.

5. Bodei L, Cremonesi M, Ferrari M, Pacifici M, Grana CM, Bartolomei M, et al. Long-term evaluation of renal toxicity after peptide receptor radionuclide therapy with 90 Y-DOTATOC and 177 LU-DOTATATE: the role of associated risk factors. Eur J Nucl Med Mol Imaging. 2008;35(10):1847-56.

6. Cives M, Strosberg J. Radionuclide therapy for neuroendocrine tumors. Curr Oncol Rep. 2017;19(2):9.

7. Strosberg JR, Wolin EM, Chasen B, Kulke MH, Bushnell DL, Caplin ME, et al. NETTER-1 phase III: progression-free survival, radiographic response, and preliminary overall survival results in patients with midgut neuroendocrine tumors treated with 177-Lu-Dotatate. American Society of Clinical Oncology; 2016.

8. Strosberg J, El-Haddad G, Wolin E, Hendifar A, Yao J, Chasen B, et al. Phase 3 trial of 177Lu-Dotatate for midgut neuroendocrine tumors. N Engl J Med. 2017;376(2):125-35.

9. Ilan E, Sandström M, Wassberg C, Sundin A, Garske- Román U, Eriksson B, et al. Dose response of pancreatic neuroendocrine tumors treated with peptide receptor radionuclide therapy using 177Lu-DOTATATE. J Nucl Med. 2015; 56(2):177-82. 
10. Bailey DL, Willowson KP. An evidence-based review of quantitative SPECT imaging and potential clinical applications. J Nucl Med. 2013;54(1):83-9.

11. Bailey DL, Willowson KP. Quantitative SPECT/CT: SPECT joins PET as a quantitative imaging modality. Eur J NuCl Med Mol Imaging. 2014;41(1):17-25.

12. Zeintl J, Vija AH, Yahil A, Hornegger J, Kuwert T. Quantitative accuracy of clinical 99mTc SPECT/CT using ordered-subset expectation maximization with 3-dimensional resolution recovery, attenuation, and scatter correction. J Nucl Med. 2010; 51(6):921.

13. Collarino A, Pereira Arias-Bouda LM, Valdés Olmos RA, van der Tol P, Dibbets-Schneider $P$, de Geus-Oei LF, et al. Experimental validation of absolute SPECT/CT quantification for response monitoring in breast cancer. Med Phys. 2018;45(5):2143-53.

14. Assie K, Dieudonné A, Gardin I, Vera P, Buvat I. A preliminary study of quantitative protocols in indium 111 SPECT using computational simulations and phantoms. IEEE Trans Nucl Sci. 2010;57(3):1096-104.

15. He B, Du Y, Song X, Segars WP, Frey EC. A Monte Carlo and physical phantom evaluation of quantitative in-111 SPECT. Phys Med Biol. 2005;50(17):4169.

16. He B, Frey EC. Comparison of conventional, model-based quantitative planar, and quantitative SPECT image processing methods for organ activity estimation using in-111 agents. Phys Med Biol. 2006:51(16):3967.

17. Green AJ, Dewhurst SE, Begent RH, Bagshawe KD, Riggs SJ. Accurate quantification of 131 I distribution by gamma camera imaging. Eur J Nucl Med. 1990;16(4-6):361-5.

18. Ljungberg M, Frey E, Sjögreen K, Liu X, Dewaraja Y, Strand S-E. 3D absorbed dose calculations based on SPECT: evaluation for 111-in/90-Y therapy using Monte Carlo simulations. Cancer Biother Radiopharm. 2003;18(1):99-107.

19. Ljungberg M, Sjögreen K, Liu X, Frey E, Dewaraja Y, Strand S-E. A 3-dimensional absorbed dose calculation method based on quantitative SPECT for radionuclide therapy: evaluation for 131I using Monte Carlo simulation. J Nucl Med. 2002:43(8):1101.

20. Beauregard J-M, Hofman MS, Pereira JM, Eu P, Hicks RJ. Quantitative 177Lu SPECT (QSPECT) imaging using a commercially available SPECT/CT system. Cancer Imaging. 2011;11(1):56.

21. Hippeläinen $E$, Tenhunen M, Mäenpää H, Sohlberg A. Quantitative accuracy of 177Lu SPECT reconstruction using different compensation methods: phantom and patient studies. EJNMMI Res. 2016;6(1):16.

22. Kangasmaa TS, Constable C, Hippeläinen E, Sohlberg AO. Multicenter evaluation of single-photon emission computed tomography quantification with third-party reconstruction software. Nucl Med Commun. 2016;37(9):983-7.

23. NM Quantification Q. Metrix for SPECT/CT Package. White Paper DOC1951185: GE Healthcare. 2017.

24. Accurate, reproducible, and standardized quantification. XSPECT Quant White Paper: Siemens Healthineers. 2018. https:// www.siemens-healthineers.com/de/molecular-imaging/trends-innovations/theranostics\#White_paper.

25. Peters SMB, van der Werf NR, Segbers M, van FHP V, Wierts R, JAK B, Konijnenberg MW, Lazarenko SV, Visser EP, Martin G. Towards standardization of absolute SPECT/CT quantification: a multi-center and multi-vendor phantom study. EJNMMI Phys. 2019;6(1):29.

26. Rathke H, Giesel FL, Flechsig P, Kopka K, Mier W, Hohenfellner M, et al. Repeated 177Lu-labeled PSMA-617 radioligand therapy using treatment activities of up to 9.3 GBq. J Nucl Med. 2018;59(3):459-65.

27. Ljungberg M, Celler A, Konijnenberg MW, Eckerman KF, Dewaraja YK, Sjogreen-Gleisner K. MIRD pamphlet no. 26: joint EANM/MIRD guidelines for quantitative 177Lu SPECT applied for dosimetry of radiopharmaceutical therapy. J Nucl Med. 2016:57(1):151-62

28. Medicine DSoN. Procedure Guidelines Nuclear Medicine. Part IV: Equipment: Kloosterhof Neer BV; 2016. p. 662-70.

29. Lowekamp BC, Chen DT, Ibáñez L, Blezek D. The design of SimplelTK. Front Neuroinform. 2013;7:45.

30. Yaniv Z, Lowekamp BC, Johnson HJ, Beare R. SimplelTK image-analysis notebooks: a collaborative environment for education and reproducible research. J Digit Imaging. 2018;31(3):290-303.

31. Frings $\mathrm{V}$, de Langen AJ, Smit EF, van Velden FH, Hoekstra OS, van Tinteren $H$, et al. Repeatability of metabolically active volume measurements with 18F-FDG and 18F-FLT PET in non-small cell lung cancer. J Nucl Med. 2010;51(12):1870-7.

32. Gear JI, Cox MG, Gustafsson J, Gleisner KS, Murray I, Glatting G, et al. EANM practical guidance on uncertainty analysis for molecular radiotherapy absorbed dose calculations. Eur J Nucl Med Mol Imaging. 2018;45(13):2456-74.

33. Cheng L, Hobbs RF, Segars PW, Sgouros G, Frey EC. Improved dose-volume histogram estimates for radiopharmaceutical therapy by optimizing quantitative SPECT reconstruction parameters. Phys Med Biol. 2013;58(11):3631.

34. Lyra M, Ploussi A. Filtering in SPECT image reconstruction. J Biomed Imaging. 2011;2011:10.

35. Dewaraja YK, Frey EC, Sgouros G, Brill AB, Roberson P, Zanzonico PB, et al. MIRD pamphlet no. 23: quantitative SPECT for patient-specific 3-dimensional dosimetry in internal radionuclide therapy. J Nucl Med. 2012;53(8):1310-25.

36. Cheebsumon P, Yaqub M, van Velden FH, Hoekstra OS, Lammertsma AA, Boellaard R. Impact of [18 F] FDG PET imaging parameters on automatic tumour delineation: need for improved tumour delineation methodology. Eur J Nucl Med Mol Imaging. 2011;38(12):2136-44

37. Frings V, van Velden FH, Velasquez LM, Hayes W, van de Ven PM, Hoekstra OS, et al. Repeatability of metabolically active tumor volume measurements with FDG PET/CT in advanced gastrointestinal malignancies: a multicenter study. Radiology. 2014;273(2):539-48.

38. Kaalep A, Sera T, Rijnsdorp S, Yaqub M, Talsma A, Lodge MA, et al. Feasibility of state of the art PET/CT systems performance harmonisation. Eur J Nucl Med Mol Imaging. 2018;45(8):1344-61.

39. Ryu H, Meikle SR, Willowson K, Eslick EM, Bailey DL. Performance evaluation of quantitative SPECT/CT using NEMA NU 2 PET methodology. Phys Med Biol. 2019;64(14):145017.

40. Zeng GL. Gibbs artifact reduction by nonnegativity constraint. J Nucl Med Technology. 2011;39(3):213-9.

41. Tran-Gia J, Lassmann M. Optimizing image quantification for 177Lu SPECT/CT based on a 3D printed 2-compartment kidney phantom. J Nucl Med. 2018;59(4):616-24.

42. Boellaard R, Delgado-Bolton R, Oyen WJ, Giammarile F, Tatsch K, Eschner W, et al. FDG PET/CT: EANM procedure guidelines for tumour imaging: version 2.0. Eur J Nucl Med Mol Imaging. 2015;42(2):328-54.

\section{Publisher's Note}

Springer Nature remains neutral with regard to jurisdictional claims in published maps and institutional affiliations. 